\title{
UN SPILOTYLENCHUS NOUVEAU \\ (Nematodea, Tylenchida : Allantonematidae) parasite de Spilopsyllus cuniculi (Dale) (Siphonaptera: Pulicidae), Siphonaptère inféodé au lapin de garenne (1)
}

\author{
H. LAUNAY* et J. DEUNFF**
}

RÉSUMÉ. Spilotylenchus beaucournui sp. n. (Nematodea, Tylenchida: Allantonematidae) parasite du Siphonaptère Spilotylenchus cuniculi (Dale), inféodé au lapin de garenne, est décrit et comparé aux autres espèces appartenant au genre Spilotylenchus. Ce nématode provoque un blocage de l'oogenèse chez les puces femelles.

\section{Spilotylenchus beaucournui sp.n. (Nematodea, Tylenchida: Allantonema- tidae) parasite of the European rabbit flea Spilopsyllus cuniculi (Dale)}

SUMMARY. Spilotylenchus beaucournui sp. n. is described and compared with the other species belonging to the genus Spilotylenchus. This nematode produce a locking of the oogenesis in female fleas.

Dans le cadre des recherches sur les possibilités prophylactiques de la lutte antivectorielle contre la myxomatose du lapin de garenne, nous avions décrit (Launay, Deunff et Bain, 1983) un nématode Allantonematidae (Tylenchida) parasite du plus répandu des Siphonaptères du lapin, Spilopsyllus cuniculi. Ce nématode, Spilotylenchus arthuri, en "castrant " les puces femelles, présente potentiellement un intérêt dans la lutte biologique. Poursuivant cette enquête, nous décrivons ici un Allantonematidae, nouveau également, spécifique de Spilopsyllus cuniculi et provoquant un blocage de l'oogenèse chez les puces femelles.

Nous sommes heureux de dédier ce taxon au Professeur J. C. Beaucournu en hommage à ses recherches sur les Siphonaptères, et, en témoignage de notre profond respect.

Nous ne reviendrons pas ici sur les méthodes d'étude qui ont été largement développées dans la publication précitée.

I. Travail effectué grâce à l'aide financière de l'A.N.C.L.A.T.R.A. et à des subventions de l'Office National de la Chasse.

* Institut de Parasitologie de l'Ouest, Faculté de Médecine, avenue du Professeur Léon-Bernard, F 35043 Rennes Cedex.

** Laboratoire de Parasitologie (Entomologie médicale) (même adresse).

Accepté le 22 novembre 1983 . 


\section{I. - Spilotylenchus beaucournui, sp. n.}

\section{1 - Matériel}

- Holotype : femelle parasite mature

- Allotype : mâle adulte libre

- Paratypes : 33 femelles parasites matures, 2 mâles adultes libres, 24 femelles adultes libres.

Tous ces spécimens obtenus à partir de Spilopsyllus cuniculi en provenance du Cap Fréhel (Côtes-du-Nord, France).

Holotype, allotype et paratypes sont déposés dans les collections du laboratoire de Parasitologie (Entomologie médicale) de la Faculté de Médecine de Rennes. L'holotype, l'allotype et une femelle adulte libre paratype seront ultérieurement déposés au laboratoire des Vers du Muséum National d'Histoire Naturelle de Paris.

\section{2 - Description}

a) Femelles parasites gravides ( $f$ ig. 8 et 9)

Longueur totale : moyenne $1266 \mu \mathrm{m}$ (925 à 1835$)$, Déviation Standard 194, holotype : $965 \mu \mathrm{m}$. Largeur maxima : $93 \mu \mathrm{m}$ (61 à 164), D. S. $=19$, holotype : $63 \mu \mathrm{m}$. Distance de l'apex au pore excréteur : $23 \mu \mathrm{m}$ (14 à 32), D. S. $=6$, holotype : $16 \mu \mathrm{m}$. Longueur de la queue $16 \mu \mathrm{m}$ (9 à 24$), \mathrm{D}$. S. $=5$, holotype : $17 \mu \mathrm{m}$; queue conique, mucron bien marqué. Distance de la vulve à l'extrémité postérieure : $48 \mu \mathrm{m}$ (34 à 68 ), D. S. $=8$, holotype : $43 \mu \mathrm{m}$; vulve sans lèvres. In vivo, couleur blanchâtre, cuticule lisse sauf dans la zone circum-orale souvent plissée, de forme arrondie. L'ovaire est bien étalé et réfléchi le plus souvent deux fois sur lui-même, la spermathèque est ovoïde ; rapport longueur de l'utérus sur longueur totale : $0,61(0,48$ à 0,61$), \mathrm{D}$. S. = 0,07 , holotype : 0,52 . Stylet fin, à ouverture ventrale non terminale, en forme de flèche, avec de légers renflements symétriques à sa base (fig. 10), longueur : 12,5 $\mu \mathrm{m}$ (11 à $13,5)$, D. S. $=0,7$, holotype : $12 \mu \mathrm{m}$.

FIG. I-IO. - Spilotylenchus beaucournui sp. $\mathrm{n}$.

I, 3 et 6 : mâle adulte libre allotype. I : vue générale latérale droite ; $3:$ partie postérieure, vue latérale droite; $6:$ partie antérieure, id.

4 : mâle adulte libre paratype, partie postérieure, vue ventrale.

2 , s et $7:$ femelle adulte libre, paratype. $2:$ vue générale latérale droite ; $5:$ partie postérieure, vue latérale droite; $7:$ partie antérieure, id.

8 et 9 : femelle parasite holotype. 9 : vue générale latérale droite ; $8:$ partie antérieure, vue latérale droite.

ro: femelle parasite paratype, stylet en vue latérale droite. 
SPILOTYLENCHUS BEAUCOURNU n. sp.

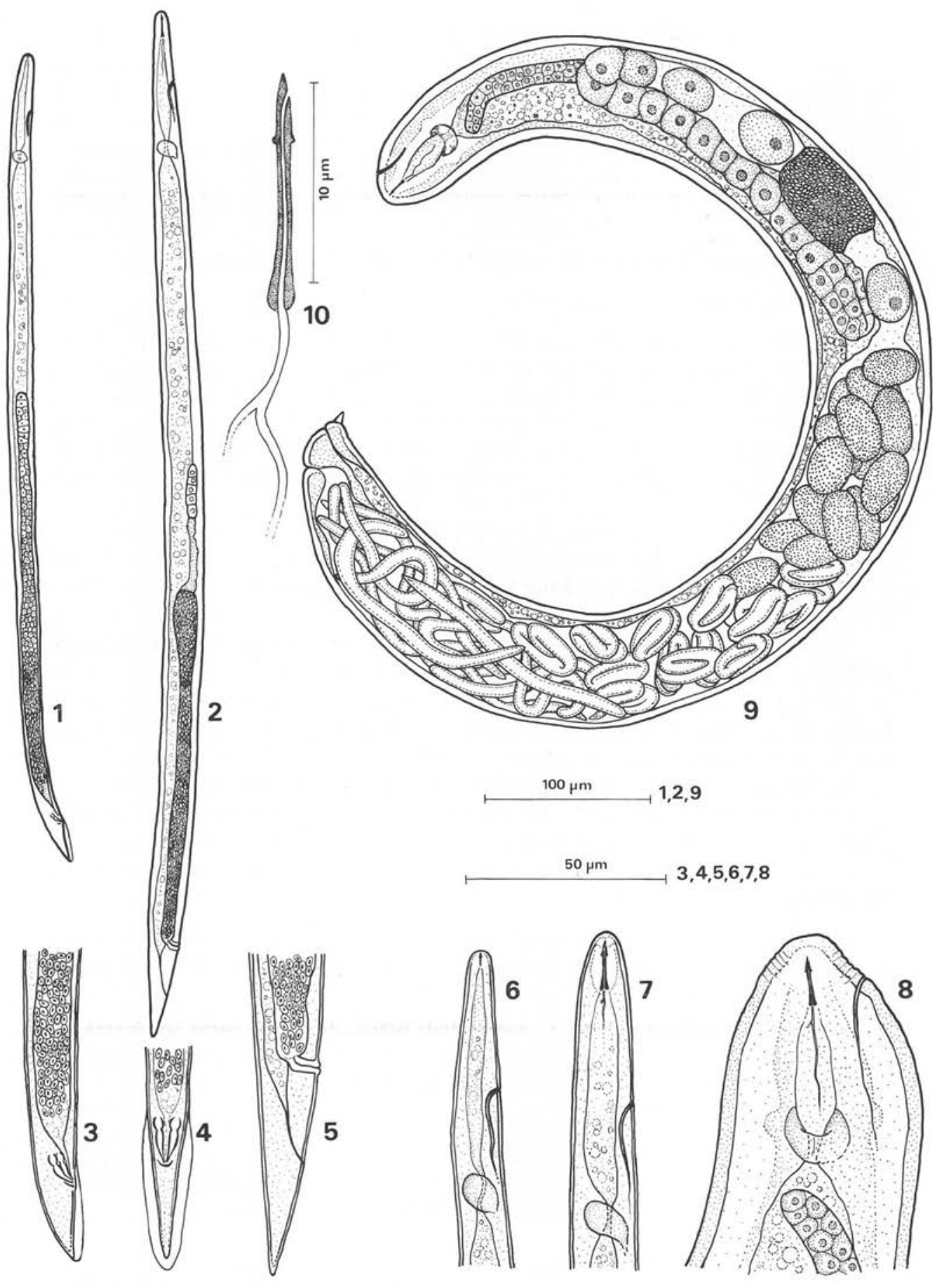


b) Femelles adultes libres ( fig. 2, 5 et 7)

Longueur totale : $595 \mu \mathrm{m}$ (515 à 685), D. S. = 50. Largeur maxima : $21 \mu \mathrm{m}$ (16 à 33), D. S. = 4. Distance de l'apex au pore excréteur : $40 \mu \mathrm{m}$ (34 à 51), D. S. $=4$. Distance de l'apex à l'anneau nerveux : $70 \mu \mathrm{m}$ (60 à 78), D. S. $=4$. Longueur de la queue : $27 \mu \mathrm{m}$ (24 à 31), D. S. = 2. Distance de la vulve à l'extrémité postérieure : $50 \mu \mathrm{m}$ (45 à 56), D. S. = 3. Cuticule lisse, même stylet que celui de la femelle parasite. En général, 5 oocytes à l'ovaire, oviducte étroit, rectiligne, spermathèque indifférenciée, utérus rectiligne à cellules indistinctes, vagin perpendiculaire à la paroi du corps, vulve avec des lèvres bien marquées et à ouverture transversale.

c) Mâles adultes libres (fig. 1, 3, 4 et 6)

Longueur totale 507 à $560 \mu \mathrm{m}$, allotype : $507 \mu \mathrm{m}$. Largeur maxima : 11 à $16 \mu \mathrm{m}$, allotype : $16 \mu \mathrm{m}$. Distance de l'apex au pore excréteur : 35 à $45 \mu \mathrm{m}$, allotype : $35 \mu \mathrm{m}$. Distance de l'apex à l'anneau nerveux : 51 à $71 \mu \mathrm{m}$, allotype : $51 \mu \mathrm{m}$. Distance de l'extrémité des spicules jusqu'à la pointe supérieure : 20 à $81 \mu \mathrm{m}$, allotype : $20 \mu \mathrm{m}$. Spicules arqués, longs de 10 à $12 \mu \mathrm{m}$, Gubernaculum réduit ( 2 à $3 \mu \mathrm{m})$. Bursa pélodère, longue de 25 à $40 \mu \mathrm{m}$ (allotype : $25 \mu \mathrm{m}$ ). Cuticule lisse, stylet très réduit, en forme de flèche, long de $4 \mu \mathrm{m}$.

\section{II. - Discussion et diagnose}

Nous rangeons ce nouveau taxon dans le genre Spilotylenchus Launay, Deunff et Bain ; il en présente toutes les caractéristiques génériques :

— une seule génération de femelles, possédant un stylet, les femelles parasites étant ovovivipares, arquées dorsalement, à maturité leur corps est occupé en majorité par l'utérus renfermant une multitude de petites larves;

- chez l'insecte on ne trouve que des femelles parasites et des larves;

— les femelles libres sont seules infestantes, leur ovaire est composé de quelques cellules alignées, la vulve est postérieure;

- les mâles libres présentent un petit stylet, deux spicules arqués, le gubernaculum est présent, la bursa pélodère.

Spilotylenchus beaucournui se distingue de S. arthuri Launay, Deunff et Bain, S. megabothridis (Laumond et Beaucournu) et $S$. laplandicus (Rubtzov et Darsakaya) par les caractères suivants :

- la taille et la forme du stylet dans les deux sexes,

- la morphologie de la gonade et des voies génitales chez les femelles parasites,

- la longueur des spicules et du gubernaculum chez les mâles.

\section{1 - Femelles adultes libres et parasites}

Les femelles adultes de $S$. beaucournui se séparent des femelles des différentes espèces regroupées dans le genre par la taille du stylet (tableau I). 
TABleau I. - Comparaison de différentes mensurations chez les femelles parasites matures et les femelles adultes libres de Spilotylenchus arthuri, beaucournui, megabothridis et laplandicus.

\begin{tabular}{|c|c|c|c|c|c|}
\hline \multicolumn{2}{|c|}{ Longueur du stylet } & $\begin{array}{c}\text { arthuri } \\
24 \text { à } 26 \mu \mathrm{m}\end{array}$ & $\begin{array}{c}\text { beaucournui } \\
11 \text { à } 13,5 \mu \mathrm{m}\end{array}$ & $\begin{array}{l}\text { megabothridis } \\
21 \text { à } 24 \mu \mathrm{m}\end{array}$ & $\begin{array}{l}\text { laplandicus } \\
19 \text { à } 22 \mu \mathrm{m}\end{array}$ \\
\hline \multirow{3}{*}{ 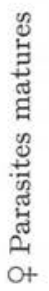 } & Longueur totale & \multicolumn{2}{|c|}{ moy. $1127 \mu \mathrm{m}$ moy. $1266 \mu \mathrm{m}$} & moy. $1119 \mu \mathrm{m}$ & 1320 à $1550 \mu \mathrm{m}$ \\
\hline & $\begin{array}{l}\text { Rapport long. de } \\
\text { l'utérus sur longueur } \\
\text { totale }\end{array}$ & moy. 0,72 & moy. 0,61 & moy. 0,65 & inf. à 0,50 \\
\hline & Hote & $\begin{array}{l}\text { Spilopsyllus } \\
\text { cuniculi }\end{array}$ & $\begin{array}{l}\text { Spilopsyllus } \\
\text { cuniculi }\end{array}$ & $\begin{array}{l}\text { Megabothris } \\
\text { turbidus }\end{array}$ & $\begin{array}{l}\text { Megabothris } \\
\text { rectangulatus }\end{array}$ \\
\hline y & Longueur totale & moy. $661 \mu \mathrm{m}$ & moy. $595 \mu \mathrm{m}$ & moy. $756 \mu \mathrm{m}$ & 700 à $800 \mu \mathrm{m}$ \\
\hline$\stackrel{5}{=}$ & $\begin{array}{l}\text { Distance de l'extré- } \\
\text { mité antérieure à } \\
\text { l'anneau nerveux }\end{array}$ & moy. $71 \mu \mathrm{m}$ & moy. $70 \mu \mathrm{m}$ & 90 à $95 \mu \mathrm{m}$ & - \\
\hline $\begin{array}{l}\text { Z̃ } \\
\text { ot } \\
\text { ot }\end{array}$ & $\begin{array}{l}\text { Distance de la vulve } \\
\text { à l'extrémité } \\
\text { postérieure }\end{array}$ & moy. $65 \mu \mathrm{m}$ & moy. $50 \mu \mathrm{m}$ & moy. $80 \mu \mathrm{m}$ & - \\
\hline
\end{tabular}

En ce qui concerne les femelles parasites matures des deux espèces parasites de Spilopsyllus cuniculi, il convient de souligner qu'elles se séparent bien morphologiquement, surtout au niveau du stylet bien sûr, mais aussi dans leur aspect général : d'une part arthuri (proche de megabothridis) qui apparaît plus grêle, moins courbée, avec un utérus énorme rejetant la spermathèque bien individualisée, sphérique, et l'ovaire, pelotonné, en avant, d'autre part beaucournui (laplandicus s'en rapproche) plus trapue, très courbée, avec un utérus moins long laissant l'ovaire bien étalé dans la partie antérieure avec une spermathèque ovoïde suivie d'un oviducte distinct qui l'éloigne de l'utérus.

Nous proposons une clé de détermination des femelles parasites matures des différentes espèces regroupées dans le genre Spilotylenchus :

A - Stylet long de 11 à $13,5 \mu \mathrm{m}$, parasite de S. cuniculi ...... beaucournui sp. $n$.

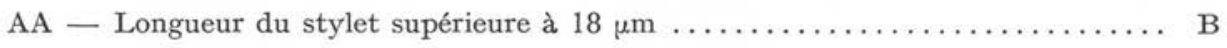

B - Longueur du corps supérieure à $1300 \mu \mathrm{m}$, stylet long de 19 à $22 \mu \mathrm{m}$, parasite de Megabothris rectangulatus ............ laplandicus (Rubtzov et Darskaya)

$\mathrm{BB}$ - Longueur du corps inférieure à $1300 \mu \mathrm{m}$, stylet long de 21 à $26 \mu \mathrm{m} \ldots \ldots$. C

C - Stylet long de 21 à $24 \mu \mathrm{m}$, asymétrique dans sa partie postérieure, parasite de Megabothris turbidus ............. megabothridis (Laumond et Beaucournu)

CC - Stylet long de 24 à $26 \mu \mathrm{m}$, symétrique dans sa partie postérieure, parasite de S. cuniculi ......................... arthuri Launay, Deunff et Bain 


\section{2 - Mâles adultes libres}

Les mâles de Spilotylenchus arthuri, beaucournui et megabothridis (les autres ne sont pas connus) sont immédiatement séparables par la longueur du stylet (tableau $I I$ ).

TABLEAU II. - Comparaison de différentes mensurations chez les mâles adultes de Spilotylenchus arthuri, beaucournui et megabothridis.

\begin{tabular}{lccc}
\hline & arthuri & beaucournui & megabothridis \\
\hline Longueur du stylet & 7 à $8 \mu \mathrm{m}$ & $4 \mu \mathrm{m}$ & 10 à $12 \mu \mathrm{m}$ \\
Longueur totale & moy. $585 \mu \mathrm{m}$ & 507 à $560 \mu \mathrm{m}$ & moy. $743 \mu \mathrm{m}$ \\
$\begin{array}{l}\text { Distance de l'extrémité } \\
\text { antérieure de l'anneau nerveux }\end{array}$ & moy. $57 \mu \mathrm{m}$ & 51 à $71 \mu \mathrm{m}$ & 90 à $95 \mu \mathrm{m}$ \\
Longueur des spicules & 14 à $18 \mu \mathrm{m}$ & 10 à $12 \mu \mathrm{m}$ & 12 à $14 \mu \mathrm{m}$ \\
Longueur du Gubernaculum & 3 à $4 \mu \mathrm{m}$ & 2 à $3 \mu \mathrm{m}$ & 8 à $9 \mu \mathrm{m}$ \\
Longueur de la queue & moy. $34 \mu \mathrm{m}$ & 20 à $31 \mu \mathrm{m}$ & moy. $43 \mu \mathrm{m}$ \\
\hline
\end{tabular}

La longueur du corps, la distance de l'extrémité antérieure à l'anneau nerveux (comme chez les femelles), le gubernaculum, la queue, sont plus grands chez megabothridis; les spicules par contre sont plus courts que ceux d'arthuri mais plus longs que ceux de beaucourmui qui présente par ailleurs les plus petites dimensions pour tous les caractères précédemment cités.

\section{BIBLIOGRAPHIE}

Laumond C., Beaucournu J. C. : Neoparasitylenchus megabothridis n. sp. (Tylenchida: Allantonematidae) parasite de Megabothris turbidus (Siphonaptera : Ceratophyllidae); observations sur les Tylenchides de puces dans le sud-ouest de l'Europe. Ann. Parasitol. Hum. Comp., $1976,53,291-302$.

LAUNAY H., DEUNFF J., BAIN O. : Spilotylenchus arthuri, gen. n., sp. n. (Nematodea, Tylenchida : Allantonematidae), parasite de Spilopsyllus cuniculi (Dale, I878) (Siphonaptera : Pulicidae). Ann. Parasitol. Hum. Comp., 1983, ,8, I4 I-150.

Rubtzov I. A. : (Parasites et ennemis des puces). Parazity i vragi blokh “Nauke”, Leningrad, USSR, I981, 104 p. 